\title{
CHEBYSHEV CONSTANT FOR CENTERED SETS
}

\author{
SUSAN L. FRIEDMAN
}

ABSTRACT. Using $\lambda$ th power means in the case $\lambda \geq 1$ it is proven that the Chebyshev constant of any bounded centered set in a metric space is equal to one-half the topological diameter of the set. Thus the Chebyshev constant for any unit ball of a normed linear space is equal to one for the above means.

1. Introduction. Let $(X, \rho)$ be a metric space and let $V$ be any bounded subset of $X$. We consider the following set functions:

and

$$
\tau_{m}^{(\lambda)}(V)=\inf _{x_{i} \in X} \sup _{x \in V^{\prime}}\left[\frac{1}{m} \sum_{i=1}^{m} \rho\left(x, x_{i}\right)^{\lambda}\right]^{1 / \lambda}, \quad \lambda \neq 0,
$$

$$
d_{m}^{(\lambda)}(V)=\sup _{x_{i}, x_{j} \in V}\left[\frac{1}{\left(\begin{array}{c}
m \\
2
\end{array}\right)} \sum_{1 \leq i<j \leq m} \rho\left(x_{i}, x_{j}\right)^{\lambda}\right]^{1 / \lambda}, \quad \lambda \neq 0 .
$$

It can be shown (see Hille [5]) that both $\lim _{m \rightarrow \infty} \tau_{m}^{(\lambda)}(V)$ and $\lim _{m \rightarrow \infty} d_{m}^{(\Lambda)}(V)$ exist. The former limit is called the $\lambda$ th power average Chebyshev constant for $V$ and denoted by $\chi^{(\lambda)}(V)$ and the latter limit is called the $\lambda$ th power average transfinite diameter for $V$ and denoted by $\delta^{(\lambda)}(V)$.

We note that these definitions involve a choice of metric space, subset, and averaging process. Selections of these have led to several papers, among which one must include the work of Fekete [1], Pólya and Szegö [8], and Hille [5]. Fekete originally defined these concepts in 1923 for compact sets $E$ in the complex plane $\mathbf{C}$ using a geometric averaging process, 1

and

$$
\tau_{m}^{(0)}(E)=\min _{z_{i} \in \mathbf{C}} \max _{\boldsymbol{z} \in E}\left[\prod_{i=1}^{m}\left|z-z_{i}\right|\right]^{1 / m}
$$

$$
d_{m}^{(0)}(E)=\max _{z_{i}, z_{j} \in E}\left(\prod_{1 \leq i<j \leq m}\left|z_{i}-z_{j}\right|\right)^{1 /\left(\begin{array}{c}
m \\
2
\end{array}\right)}
$$

where $\chi^{(0)}(E)=\lim _{m \rightarrow \infty} \tau_{m}^{(0)}(E)$ and $\delta^{0}(E)=\lim _{m \rightarrow \infty} d_{m}^{(0)}(E)$. Fekete then

Received by the editors November 12, 1973 and, in revised form, May 21, 1974. AMS (MOS) subject classifications (1970). Primary 52-00; Secondary 52A40.

1 The superscript 0 is used since it can be shown that these means result from the above in the limit as $\lambda \rightarrow 0$. 
proved that for any compact set $E$ in the complex plane $\chi^{(0)}(E)=\delta^{(0)}(E)$.

Pólya and Szegö considered certain compact sets in $R_{1}, R_{2}$, and $R_{3}$ ( $R_{n}$ being real Euclidean $n$-space) and calculated their transfinite diameters and Chebyshev constants for $\lambda$ th power averages. Hille considered more generalized averaging processes and extended the work to certain subsets of function spaces. A summary of his results can be found in the last chapter of his recent book [4]. He states there that the calculation of the Chebyshev constant for unit balls in Banach spaces of infinite dimension does not seem to have been attacked.

In this paper we will make the calculation for a certain class of averaging processes for the unit ball in any normed linear space. In fact it will be shown that for bounded centered sets $V$ in a metric space, the Chebyshev constant for $\lambda$ th power averages, $\lambda \geq 1$, is equal to one-half the topological diameter of the set. For a large class of Banach spaces this value is equal to the radius of the "spanning sphere" of the set. This result extends a similar one dealing with real Euclidean $n$-space in a previously published paper by the author [2].

2. Centered sets and spaces. The concept of centered sets and spaces is introduced by Kolmogorov and Tihomirov [7].

Definition. Let $(X, \rho)$ be a metric space. A bounded subset $V$ of $X$ of topological diameter $d(V)$ is said to be a centered set if there exists a point $x_{V} \in X$ such that $\rho\left(v, x_{V}\right) \leq d(V) / 2$ for all $v \in V$.

A point $x_{V}$ with this property will be called a center for $V$. We note that a center for $V$ does not have to lie in $V$.

Definition. If every bounded subset of a metric space $(X, \rho)$ is a centered set, then we shall say $X$ is a centered space.

It is interesting to note which familiar spaces are centered and which are not.

Theorem (Kolmogorov and Tihomirov). The space $R(X)$ of real-valued functions defined on an arbitrary set $X$ with the metric $\rho(f, q)=$ $\sup _{x \in X}|f(x)-g(x)|$ is centered.

The proof of this theorem may be found in [7]. It is shown there that if we define $f_{0}(x)=1 / 2[\bar{f}(x)+\underline{f}(x)]$ where $\bar{f}(x)=\sup _{f \in V} f(x)$ and $\underline{f}(x)=$ inf $_{f \in V} f(x)$ and $V$ is a bounded subset of $R(X)$, then $f_{0}(x)$ satisfies the definition of center.

The previous theorem holds for $C[a, b]$ with its usual norm, and $L^{\infty}(X)$. However we show that consideration of complex-valued functions does not lead to a centered space. 
Theorem 1. The space $C(X)$ of complex-valued functions defined on an arbitrary set $X$ with sup norm is not a centered space.

Proof. Suppose $V=\left\{f_{1}(z), f_{2}(z), f_{3}(z)\right\}$ such that $f_{i}(z)=c_{i}, i=1,2,3$ for all $z \in X$, an arbitrary set in $C$. Suppose further that $c_{i}$ are the vertices of an equilateral triangle of side $s$. Then $V$ has no center, for if $g(z)$ were a center for $V$, then $\max _{z \in V}\left|g(z)-f_{i}(z)\right| \leq 1 / 2 s$ for $i=1,2,3$ and thus $g(z)$ must be simultaneously inside or on circles with centers at $c_{i}$ and radii equal to $1 / 2 s$. But no point having this property exists. Hence $V$ has no center and $C(X)$ is not a centered space.

The proof of the last theorem establishes also that $R_{2}$ is not a centered space. In fact:

Theorem 2. Real Euclidean $n$-space $R_{n}$ is centered if and only if $n=1$.

Proof. It can be shown (see Jung [6]) that if $E$ is a regular simplex of $n+1$ vertices of side $d(E)$ in $R_{n}$, then its circumscribing sphere has radius $\sqrt{n / 2(n+1)} d(E)$. For $n>1$, this is greater than $d(E) / 2$. Hence no center can exist. When $n=1$ we have equality.

Theorem 3. The space $R_{n}^{\infty}$ (n-tuples with metric

$$
\left.\rho(x, y)=\max \left(\left|x^{(1)}-y^{(1)}\right|,\left|x^{(2)}-y^{(2)}\right|, \cdots,\left|x^{(n)}-y^{(n)}\right|\right)\right)
$$

is centered.

Proof. Let $V$ be a bounded subset of $R_{n}^{\infty}$.

$$
\begin{aligned}
d(V) & =\sup _{v_{i}, v_{j} \in V}\left\|v_{i}-v_{j}\right\| \\
& =\sup _{v_{i}, v_{j} \in V} \max \left(\left|x_{i}^{(1)}-x_{j}^{(1)}\right|,\left|x_{i}^{(2)}-x_{j}^{(2)}\right|, \cdots,\left|x_{i}^{(n)}-x_{j}^{(n)}\right|\right) \\
& =\max \left(\sup _{v_{i}, v_{j} \in V}\left|x_{i}^{(1)}-x_{j}^{(1)}\right|,\left|x_{i}^{(2)}-x_{j}^{(2)}\right|, \cdots,\left|x_{i}^{(n)}-x_{j}^{(n)}\right|\right) .
\end{aligned}
$$

Consider an $n$-dimensional cube $S$ of side $d(V)$. Then from the boundedness of $V$, such a cube will enclose $V$. Let the boundary of $S$ be denoted by $\widetilde{S}$. Choose any $v \in V=\left(v^{(1)}, v^{(2)}, \ldots, v^{(n)}\right)$. Now

$$
\begin{aligned}
\rho(v, \tilde{S}) & =\min _{x \in \tilde{S}}\|v-x\| \\
& =\min _{x \in \tilde{S}} \max \left(\left|v^{(1)}-x^{(1)}\right|,\left|v^{(2)}-x^{(2)}\right|, \ldots,\left|v^{(n)}-x^{(n)}\right|\right) .
\end{aligned}
$$

There exist points $x_{1} \in \tilde{S}$ and $x_{2} \in \tilde{S}$ such that $s_{1}$ and $s_{2}$ are on oppo- 
site faces of $S$. Let $x_{1}=\left(v^{(1)}, v^{(2)}, \ldots, w^{(i)}, \ldots, v^{(n)}\right)$ and $s_{2}=\left(v^{(1)}\right.$, $\left.v^{(2)}, \ldots, u^{(i)}, \ldots, v^{(n)}\right)$. Then $\rho\left(s_{1}, s_{2}\right)=\left|w^{(i)}-u^{(i)}\right|=d(V)$. But $\rho\left(v, s_{1}\right)=$ $\left|v^{(i)}-w^{(i)}\right|$ and $\rho\left(v, s_{2}\right)=\left|v^{(i)}-u^{(i)}\right|$. Since $\left|v^{(i)}-w^{(i)}\right|+\left|v^{(i)}-u^{(i)}\right|=$ $\left|w^{(i)}-u^{(i)}\right|=d(V)$, at least one of $\rho\left(v, s_{1}\right), \rho\left(v, s_{2}\right)$ must be less than $d(V) / 2$. Hence

$$
\rho(v, s)=\min _{x \in \tilde{S}} \rho(v, x) \leq \min \left\{\rho\left(v, s_{1}\right), \rho\left(v, s_{2}\right)\right\} \leq d(V) / 2 .
$$

Thus the center of $S$ serves as a center for $V$.

For normed linear spaces $X$ it is customary to define a ball $B$ of radius $R$ with center at $c$ as $B=\{x \in X \mid\|x-c\| \leq R\}$.

Theorem 4. Any ball of a normed linear space $X$ is centered.

Proof. Now $d(B)=\sup _{x, y \in B}\|x-y\|$. Since $\|x-y\| \leq\|x-c\|+\|c-y\|$ for all $x$ and $y$ we have $\sup _{x, y \in B}\|x-y\| \leq \sup _{x, y \in B}\{\|x-c\|+\|c-y\|\}$ or $d(B) \leq 2 R$. If $x \in B$, then $(-x+2 c) \in B$, and $\|x-(-x+2 c)\|=2 R$. But $\sup _{x, y \in B}\|x-y\| \geq\|x-(-x+2 c)\|$. Hence $d(B) \geq 2 R$. Thus $d(B) / 2=R$ and $B$ is centered.

Bearing in mind that the following theorem applies to all bounded subsets of the heretofore mentioned centered spaces as well as to any ball of a normed linear space we are now ready to prove the main theorem:

Theorem 5. Let $(X, \rho)$ be a metric space. If $W$ is a centered subset of $X$ of topological diameter $d(W)$, then $\chi^{(\lambda)}(W)=d(W) / 2$ for $\lambda \geq 1$.

Proof. $\tau_{m}^{(\lambda)}(W)=\inf _{x_{i} \in X} \sup _{w \in W}\left[m^{-1} \Sigma_{i=1}^{m} \rho\left(w, x_{i}\right)^{\lambda}\right]^{1 / \lambda}$. Let $x_{W} \in X$ be a center for $W$. Set $x_{i}^{i}=x_{W}$ for $i=1,2, \ldots, m$. Then

$$
\tau_{m}^{(\lambda)}(W) \leq \sup _{w \in W}\left[\frac{1}{m} m \rho\left(w, x_{W}\right)^{\lambda}\right]^{1 / \lambda}=\sup _{w \in W} \rho\left(w, x_{W}\right) \leq \frac{d(W)}{2} .
$$

If $W$ contains only one point, both $d(W)$ and $\chi^{(\lambda)}(W)$ are zero and the theorem is proved. Hence we suppose $W$ contains more than one point. Let $\bar{w}$ and $\underline{w}$ be two distinct elements of $W$. For every $\epsilon>0$, there exists $\bar{x}_{1}$. $\bar{x}_{2}, \ldots, \bar{x}_{m} \in X$ such that

$$
\sup _{w \in W}\left[\frac{1}{m} \sum_{i=1}^{m} \rho\left(w, \bar{x}_{i}\right)^{\lambda}\right]^{1 / \lambda}-\epsilon<\tau_{m}^{(\lambda)}(W) .
$$

The triangle inequality gives

$$
\rho(\underline{w}, \bar{w}) \leq \rho\left(\underline{w}, \bar{x}_{i}\right)+\rho\left(\bar{x}_{i}, \bar{w}\right) \quad \text { for } i=1,2, \cdots, m .
$$


Hence

$$
m[\rho(\underline{w}, \bar{w})]^{\lambda} \leq \sum_{i=1}^{m}\left[\rho\left(\underline{w}, \bar{x}_{i}\right)+\rho\left(\bar{x}_{i}, \bar{w}\right)\right]^{\lambda} .
$$

Raising to the $(1 / \lambda)$ th power, using Minkowski's inequality, and dividing through gives

$$
\rho(\underline{u}, \bar{w}) \leq\left[\frac{1}{m} \sum_{i=1}^{m} \rho\left(\underline{w}, \bar{x}_{i}\right)^{\lambda}\right]^{1 / \lambda}+\left[\frac{1}{m} \sum_{i=1}^{m} \rho\left(\bar{w}, \bar{x}_{i}\right)^{\lambda}\right]^{1 / \lambda} .
$$

Now, taking the supremum over all pairs $\underline{w}, \bar{w}$ in $W$ we have

$$
d(W) \leq 2 \sup _{w \in W}\left[\frac{1}{m} \sum_{i=1}^{m} \rho\left(w, \bar{x}_{i}\right)^{\lambda}\right]^{1 / \lambda} .
$$

From $(* *)$ this gives $d(W) / 2<\tau_{m}^{(\lambda)}(W)+\epsilon$. Since $\epsilon$ was arbitrary we have $d(W) / 2 \leq \tau_{m}^{(\lambda)}(W)$ and with $(*)$ as $m \rightarrow \infty, \chi^{(\lambda)}(W)=d(W) / 2$.

Theorems 4 and 5 imply the result:

Theorem 6. For any normed linear space $X$ with unit ball $U, \chi^{(\lambda)}(U)$ $=1$ for $\lambda \geq 1$.

Definition. Let $x_{1}^{*}, x_{2}^{*}, \ldots, x_{m}^{*} \in X$ be such that

$$
\tau_{m}^{(\lambda)}(V)=\sup _{x \in V}\left[\frac{1}{m} \sum_{i=1}^{m} \rho\left(x, x_{i}^{*}\right)^{\lambda}\right]^{1 / \lambda} .
$$

Then $x_{1}^{*}, x_{2}^{*}, \ldots, x_{m}^{*}$ are called Chebyshev points of order $m$ for $V$, where $V$ is a bounded subset of the metric space $(X, \rho)$.

From the proof of Theorem 5 we see

Theorem 7. If $x_{W}$ is a center for $W$, then $x_{i}^{*}=w_{W}$ for $i=1,2, \ldots, m$ is a set of Chebyshev points of order $m$ for $W$.

\section{Chebyshev centers.}

Definition. Let $(X, \rho)$ be a metric space and $V$ a bounded subset of $X$. We examine the value $\tau_{1}(V)=\inf _{x_{1} \in X} \sup _{x \in V} \rho\left(x, x_{1}\right)$. A point $x_{i}^{*} \in X$ such that $\tau_{1}(V)=\sup _{x \in V} \rho\left(x, x_{i}^{*}\right)$ is called a Chebyshev center for $V$.

We note that a Chebyshev center for $V$ is a Chebyshev point of order one for $V$. Since an average function of one term must coincide with that term, we omit any superscript on $\tau_{1}$. Thus a Chebyshev center for $V$ is independent of the average used. This definition appears in Singer [10] in a different context, but as its name implies, it is related to the concept of center.

Theorem 8. Let $W$ be a centered subset of a metric space $(X, \rho)$. Then 
if $x_{W}$ is a center for $W, x_{W}$ is a Chebyshev center for $W$.

Proof. Suppose $x_{W}$ is not a Chebyshev center for $W$. Then $\sup _{x \in W} \rho\left(x, x_{W}\right)>\tau_{1}(W)$. Since $W$ is centered we have, from Theorem 5 , $\tau_{1}(W)=d(W) / 2$. Hence $\sup _{x \in W} \rho\left(x, x_{W}\right)>d(W) / 2$. Thus $x_{W}$ is not a center for $W$.

Theorem 9. Any Chebyshev center of a centered set $W$ in a metric space is a center.

Proof. Since $\sup _{x \in W} \rho\left(x, x_{1}^{*}\right)=\tau_{1}(W)$ and $\tau_{1}(W)=d(W) / 2$, we have $\rho\left(x, x_{1}^{*}\right) \leq d(W) / 2$ for all $x \in W$ and $x_{1}^{*}$ is a center.

We note that the hypothesis of Theorem 9 cannot be weakened to sets $V$ which are merely bounded. For example, if $V$ were an equilateral triangle, then its circumcenter is a Chebyshev center, but not a center!

In a previous paper [2], the author showed that the Chebyshev center played an important role in finite-dimensional Euclidean space. Indeed, the set of points $x_{i}=x_{1}^{*}$ for $i=1,2, \ldots, m$ is a set of Chebyshev points of order $m$ for any compact set $E$, for all $m$, where $\lambda \geq 1$. Moreover the Chebyshev constant for such sets is equal to the radius of the spanning sphere for $E$, i.e., the boundary of the $n$-dimensional ball of smallest radius which contains $E$.

In finite-dimensional Euclidean space the existence and uniqueness of Chebyshev centers for bounded sets is assured. This is not true for more general normed linear spaces. Existence and Uniqueness Theorems A and B have been given by Garkavi [3] as follows:

Theorem A. Let $X$ be a Banach space with the property that there exists a projection $p: X^{* *} \rightarrow X$ of norm one. Then for every bounded subset $V$ of $X$ there exists a Chebyshev center for $V$.

The existence of such a projection is known for Banach spaces which are equivalent to conjugate spaces. This does not characterize conjugate spaces, however, for such projections exist in spaces which are not the conjugate space of any Banach space. See Ruston [9].

It seems natural to extend the definition of spanning sphere to any bounded subset $V$ of a Banach space satisfying the hypothesis of Theorem A. If we denote such spaces by $G$, then we will define the center of a spanning sphere for a bounded set $V$ of $G$ as a Chebyshev center for $V$ and the radius $R$ of a spanning sphere as $R=\tau_{1}(V)$. With this definition Theorem 5 can be brought into line with its Euclidean counterpart: 
Theorem 10. $\chi^{(\lambda)}(W)=R$ for $\lambda \geq 1$ for all bounded, centered subsets $W$ of $G$, where $R$ is the radius of the spanning sphere for $W$.

Theorem B. In order that every bounded set $V$ in a normed linear space $X$ have at most one Chebyshev center, it is necessary and sufficient that the space $X$ be uniformly convex in every direction.

An example of a bounded set with more than one Chebyshev center is furnished by the following: Let $X=R_{2}^{\infty}$ and $V=[-1,1]$ on the $x^{(1)}$ axis. Then $\tau_{1}(V)=1$ and all points in the segment from -1 to 1 on the $x^{(2)}$ axis are Chebyshev centers for $V$. A spanning sphere of the set $V$ consists of any square with sides parallel to the $x^{(1)}$ and $x^{(2)}$ axes of length two and with center on the segment from -1 to 1 on the $x^{(2)}$ axis.

Theorems A and B imply the existence and uniqueness of the Chebyshev center for any bounded set in a Hilbert space.

\section{REFERENCES}

1. M. Fekete, Über die Verteilung der Wurzeln bei gewissen algebraischen Gleichungen mit ganzzahligen Koeffizienten, Math. Z. 17 (1923), 228-249.

2. S. L. Friedman, Chebyshev constant and Chebyshev points, Trans. Amer. Math. Soc. 186 (1973), 129-139.

3. A. L. Garkavi, The best possible net and the best possible cross-section of a set in a normed space, Izv. Akad. Nauk SSSR Ser. Mat. 26 (1962), 87-106; English transl., Amer. Math. Soc. Transl. (2) 39 (1964), 111-132. MR 25 \#429.

4. E. Hille, Methods in classical and functional analysis, Addison-Wesley, Reading, Mass., 1972.

5. - Topics in classical analysis, Lectures on Modern Math., Vol. 3, Wiley, New York, 1965, pp. 1-57. MR 31 \#2357.

6. H.W. E. Jung, Über die kleinste Kugel, die eine raumliche Figur einschliesst, J. für Math. 123 (1901), 241-257.

7. A. N. Kolmogorov and V. M. Tihomirov, $\epsilon$-entropy and $\epsilon$-capacity of sets in function spaces, Uspehi Mat. Nauk 14 (1959), no. 2 (86), 3-86; English transl., Amer. Math. Soc. Transl. (2) 17 (1961), 277-364. MR 22 \# 2890; 23 \# A2031.

8. G. Pólya and G. Szegö, Über den transfiniten Durchmesser (Kapazitatskonstante) von ebenen und raumlichen Punktmengen, J. Reine Angew. Math. 165 (1931), $4-49$.

9. A. F. Ruston, A note on the greatest crossnorm, Proc. Amer. Math. Soc. 13 (1962), 828-829. MR 26 \#6722.

10. I. Singer, Best approximation in normed linear spaces by elements of linear subspaces, Editura Academiei Republicii Socialiste România, Bucharest, 1967, English transl., Die Grundlehren der math. Wissenschaften, Band 171, Springer-Verlag, Berlin and New York, 1970. MR 38 \#3677, 42 \# 4937.

DEP ARTMENT OF MATHEMATICS, BARUCH COLLEGE (CUNY), NEW YORK, NEW YORK 10010 